\title{
Quick Ice Cream
}

\author{
Esmeralda Terrazas ${ }^{1}$ \\ ${ }^{1}$ Affiliation not available
}

June 7, 2021

June 04, 2021

Suggested time: 50 minutes

\section{Overview}

In this lesson the students will learn about the states of the reactions and how a catalyzer can help to speed up or slow down the reaction. They will put up an easy factory of ice cream to see how a specific catalyzer speed up a reaction from liquid to solid.

\section{Materials}

- Bag of ice

- 1 kilogram of grain salt

- 1 big metal bowl

- 1 medium metal bowl

- 1 box of juice/ some homemade juice

- 1 cup

- 1 spoon

\section{Introduction}

As you will see in the first link of YouTube all in the world is considered material, and every material works in three different states of the material, the states are: solid, liquid and gas, solid is every material that you can touch grab with your hand like the computer or cellphone or tablet in which you are seeing this, solid is every material that you can touch and grab, the state of liquid is easy to explain, liquids are everything that share characteristics with water and the easiest example of the liquids is the water, there are another examples of liquids like juice, soda, etc. Liquids as solids are material that you can touch with your hand but there is a difference between liquids and solids, solids are the material that you can touch but also you can grab with your hand and liquids are the material that you can touch with your hand, but you cannot grab water. Gas is the material that you cannot even see, the gases are material that you cannot touch or see, the easiest example of a gas is the air, the air is the most common gas. When you boil the water, it changes of state of the material from liquid to gas, so you cannot see gas water, but it is there. 
And as you can see in the second video a catalyzer is basically a substance that speed up a reaction, the chemical definition of a catalyzer is some chemical that accelerates a process.

A chemical process is the process that make some change in the material, when a solid change to liquid is a chemical process, when the water is boiling till evaporating it is a chemical process. So, how does this concept fit in this experiment? Well, the experiment is making ice cream without a fridge and we will do it by using a common catalyzer. Our experiment consists in having the ice in a big bowl and having the juice on a bowl smaller than the bowl with ice, but how does the concept of catalyzer is applicated to this? In the ice bowl we put a lot of grain salt, this is the catalyzer, and the salt accelerates the process of melting in the ice but between this process and the moment when there is only cold water, there is a process of reduction of the congelation point of the ice which means that the ice gets colder than its normal temperature at melting time, this extra cold is what makes the experiment successful.

\section{Multimedia Resources}

States of the material for children - What are the states of the material? Solid, Liquid and Gashttps://www.youtube.com/watch? $v=f x D K p E Y A o S E$

Science in 1': What is a catalyzer?https://www.youtube.com/watch?v=YQtUKMkUHKg

\section{Lesson Plan}

\section{Part 1: I can see reactions? (10 min)}

See the first video of the state of the material.

Explain what the three state of material are and analyze an example of every day to make a better comprehension.

\section{Part 2: Do it faster! (15 min)}

1. Propose the question if there is something that can speed up or slow down a reaction from a state to a different state of material.

2. See the video of what a catalyzer is and explain it with your words without forgetting to give examples.

3. Analyze which materials can be a catalyzer and what are not. Present in the screen 5 materials that we can find in house, the students will vote if it is or isn't a catalyzer.

\section{Part 3: Let us make ice cream without fridge! (25 min)}

1. Explain the students that the reaction we are about to make is from liquid to solid and we are going to speed it up with a catalyzer that it is going to be de grain salt.

2. For the reaction to be made we will freeze the juice to make it ice cream that is why we need the ice for.

3. Let's start to put a lot of ice in the big metal bowl. Spread the ice so that it covers all the bowl.

4. Add a big amount of grant salt to the top of the ice. Explain the reaction that is happening with the ice and the salt.

5. Then cover up the ice and the salt with the other metal bowl seeing upside up. Add the juice in the top metal bowl. 
6. Start spinning the top bowl with the juice inside 5 seconds to the left and 5 seconds to de right while you spread it through the bowl with a spoon. Repeat this for 20 min.

7. Analyze the reaction that is forming and speeded up from the catalyzer.

8. For the final product put your ice cream in a cup with some help of a spoon.

\section{After the Lesson}

Once you have your ice cream in the cup, students must clean their metal bowls. The water of the ice or the left ice can be poured out to water the plants.

\section{Enjoy your ice cream and review what the students saw of the states of materials.}

